\title{
Movement amplitude choice reaction time performance in Parkinson's disease may be independent of dopaminergic status
}

\author{
Seth L Pullman, Ray L Watts, Jorge L Juncos, Jerome N Sanes
}

\begin{abstract}
The effect of circulating levels of plasma levodopa on reaction time performance was studied in patients with Parkinson's disease and untreated normal controls when instructed to move either a shorter or longer distance. On half the movements, subjects were pre-cued on the direction and amplitude of an impending movement. On the remaining movements, only the direction was prespecified, and the amplitude was determined only when the cue to move was presented. Reaction time performance of patients was evaluated at three infusion levels of levodopa so that the patients were optimally, moderately, or minimally medicated. Parkinsonian patients were always slower to react and move than normal subjects. Clinical state correlated with movement time, but not with reaction time. These results contrast with those in which reaction time was related to plasma levodopa levels when movement direction and initiation were processed concomitantly, but the movement amplitude was precued. It is possible that specification of the amount of muscle activity is partially independent of dopaminergic transmission.
\end{abstract}

Neurological Institute, Columbia-

Presbyterian Medical Center, New.York

S L Pullman

Department of Neurology,

Emory University,

Atlanta, Georgia

R L Watts

J L Juncos

Human Motor Control Section, Medical

Neurology Branch,

National Institute of

Neurological

Disorders and Stroke,

National Institutes of

Health, Bethesda,

Maryland, United

States

J N Sanes

Correspondence to: Dr Jerome N Sanes, Center for Neural Science, Box 1953, Brown University, Providence, RI 02912 United States.

Received 19 July 1989 and in revised form 2 October 1989.
In addition to slowness of movement execution, patients with Parkinson's disease (PD) typically initiate movements slower than normal. ${ }^{1-4}$ It appears that the fundamental defect in reaction time (RT) performance in $\mathrm{PD}$ is a delay in starting simple movements for which all the parameters of movement, such as amplitude, direction, and which body part to move, are specified in advance. ${ }^{56}$ In normal subjects, $\mathrm{RT}$ is prolonged when parameters of movement before receiving the signal to move. ${ }^{78}$ These results are widely interpreted as indicating that the initiation process is slowed when concurrent decisions need to be made about the characteristics of the impending movement. Since the difference in movement time (MT) between normal subjects and patients with PD progressively increases with movement complexity, ${ }^{9}$ it might also be expected that increases in movement complexity would increasingly slow movement initiation in PD more than normal. However, PD patients, although typically slower to begin movements than subjects are uninformed about these normal subjects in all instances, do not usually show added deficits in RT with increases in movement complexity. Indeed, in some RT situations in which a behavioural choice and movement initiation are required simultaneously, PD patients can initiate movement at latencies comparable to those of normal subjects. ${ }^{410}$

A problem with many previous investigations of RT performance in PD is that the clinical state of the patients has not been rigorously controlled. The potential difficulty of this problem was overcome in our recent investigation of choice and simple RT in relation to circulating levels of levodopa. ${ }^{10}$ We found that the paradoxical result of a seemingly normal RT when PD patients performed in a directional choice RT task was related to the circulating levels of levodopa. In our study, the choice RT in PD was not different from normal and was only slightly longer than simple RT in PD when patients were optimally medicated. However, when levodopa was reduced so that the patients were in an "off" clinical state, the choice RT was sufficiently delayed to be significantly different from normal. In contrast, the simple RT was unresponsive to changes in levodopa concentration. We concluded that the specification of directional information is a dopamine sensitive process in PD and that the failure of other investigators to see prolongation of RT in PD when movement initiation and specification are required simultaneously, ${ }^{41}$ may have been related to patient selection and fluctuating levels of levodopa.

This study was designed to investigate whether the simultaneous specification of movement amplitude and initiation of movement by PD patients is affected by levodopa administration. It is known that neurons within the basal ganglia discharge in relation to the direction of intended or executed movement. ${ }^{12-14}$ Additionally, it appears that basal ganglia neurons discharge in relation to the magnitude of an opposing load; thus they are responsive to the amount of voluntary muscle activity. ${ }^{12151.6}$ It is possible that a disruption of basal ganglia function might lead to a deficit in the control of the magnitude of EMG activity. However, since PD patients seem able to scale the first agonist burst normally in relation to movement amplitude and opposing load, ${ }^{17}$ it might be expected that PD patients do not have an added deficit in $\mathrm{RT}$ when required to specify movement amplitude and initiation simultaneously. 


\section{Materials and methods}

Five right handed patients with $P D$, aged 29 to 61 years (mean, $49.2 \mathrm{yr}$ ), were selected for minimal tremor, demonstration of "wearing off" response patterns to levodopa, absence of clinical depression, and capability of following complex instructions. They were all taking a combination of levodopa/carbidopa, and their disability scores without medication ranged from II to IV on the Hoehn and Yahr scale. ${ }^{18}$ Five age-matched normal subjects, aged 31 to 64 years (mean $47.0 \mathrm{yr}$ ), were also studied. Informed consent was obtained, and all subjects participated in the study according to guidelines specified by the NIH institutional review board for human studies. The PD patients and the normal volunteers were the same as those examined in a previous study. ${ }^{10}$

\section{Clinical treatment}

The Parkinsonian signs of patients were rated independently by two neurologists using a modified Columbia scale. ${ }^{19}$ The ratings were done at clinical baseline and after stabilisation at each of three rates of levodopa infusion. Levodopa was prepared in $0.45 \%$ saline to a concentration of $2 \mathrm{mg} / \mathrm{ml}$ and given by continuous IV infusion, with oral carbidopa, $50 \mathrm{mg}$ every three hours, starting the day before testing. Infusion rates of levodopa (mgm/ $\mathrm{kgm} /$ hr) were established empirically for each patient based on the corresponding individual clinical response. Three rates (high, middle and low) were determined to achieve three Parkinsonian states (optimal or "on", mid level, and "off"). Rate adjustments were made two hours before administration of each set of movement trials to allow stabilisation of clinical performance and plasma levodopa levels. Simultaneous with the clinical ratings, blood samples were drawn and stored immediately at $-70^{\circ} \mathrm{C}$ for subsequent assay with highperformance liquid chromatography with electrochemical detection. ${ }^{20}$

\section{Apparatus and Procedures}

The subject was seated and the lower arm and hand were restrained between lightly padded supports with the elbow angle at $\sim 90^{\circ}$. The hand was restrained within a handle that was coupled to a low friction, brushless DC torque motor (Aeroflex TQ-64). The hand was positioned so that flexion-extension movements about the wrist could be performed in the horizontal plane, and the wrist joint was colinear with the axle of the torque motor. A video monitor was placed approximately $1 \mathrm{~m}$ in front of the subject. A cursor in the form of a $3 \times 3$ matrix of small spots representing the instantaneous orientation of the right hand and a target cursor in the form of a rectangular box that was $4^{\circ}$ to $8^{\circ}$ of wrist rotation from side-toside were visible on the video screen. The subject was required to position the hand to align the two cursors only with the aide of the video display, since the hand could not be seen directly. Additional details concerning the torque motor system can be found elsewhere. ${ }^{21}$ Bipolar surface electrodes were used to record the electromyogram (EMG) from wrist extensor and flexor muscles.

The subject performed a simple and choice visual RT task requiring $15^{\circ}$ or $30^{\circ}$ of wrist flexion. For the simple RT task, a single centrally located target appeared on the video screen for initial alignment at $0^{\circ}$ of wrist flexion. A preparatory target with dashed lines, located at either $15^{\circ}$ or $30^{\circ}$ of wrist flexion appeared after $1 \mathrm{~s}$. This pretarget was used by the subject to prepare the amplitude of the impending movement. After an additional 0.8 to $1.8 \mathrm{~s}$ delay, the central target disappeared and the pretarget changed appearance to become solid-lined; this was the signal to begin a flexion movement directed toward the target as soon and as rapidly as possible. For the choice RT task, the centrally located target and then two outlined preparatory targets, located at $15^{\circ}$ and $30^{\circ}$ of wrist flexion, appeared on the video screen. After a brief delay, the central and one of the pretargets disappeared and the remaining pretarget became solid lined. As before, this was the signal to begin movement. Practice sessions were given until the subject performed movements that were consistently fast and accurate. The four types of movements (simple RT to $15^{\circ}$ and $30^{\circ}$ and choice RT to $15^{\circ}$ and $30^{\circ}$ ) were performed in one block of 100 trials. The trial order presentation was pseudo random, so that an equal number of trials of each type, 25, was presented. After practice, controls performed three sets of 100 trials, and patients performed one set of trials at each infusion rate. Wrist position and the full-wave rectified and low-pass filtered $(50 \mathrm{~Hz})$ EMG were digitised at $200 \mathrm{~Hz}$ for subsequent analysis.

\section{Data analysis}

Each trial was inspected off-line using semiautomatic interactive software that allowed for marking the beginning and end of movement with an electronic cursor. RT was defined as the time from the go signal to the onset of movement velocity. Trials were rejected if RT was shorter than $175 \mathrm{~ms}$ or longer than $850 \mathrm{~ms}$. MT was measured from the beginning of movement to the first zero-cross in the velocity profile. If movements of the PD patients were hypometric, there would be zero-crosses in velocity before the hand moved the correct distance. In these cases, MT was measured as the first zero cross after the hand had moved to $3^{\circ}$ short of the correct movement amplitude. Kinematic data from each subject and for each trial type were tabulated, averaged and analysed with $t$ tests, factorial and repeated measure analyses of variance, and linear regression procedures. Unless noted, the probability levels refer to statistics from the analyses of variance.

\section{Results \\ Clinical}

The plasma levodopa concentration was positively correlated with the corresponding rate of levodopa infusion $(R=0.88$, 
Table Plasma levodopa levels and corresponding clinical scores for PD patients

\begin{tabular}{|c|c|c|}
\hline Clinical state & $\begin{array}{l}\text { Plasma levodopa level } \\
\text { in } \mu g m / m l(S E M)\end{array}$ & Clinical score (SEM) \\
\hline $\begin{array}{l}\text { High } \\
\text { Mid } \\
\text { Low }\end{array}$ & $\begin{array}{l}6.34(1.50) \\
4.30(0.96) \\
3.06(1.10)\end{array}$ & $\begin{array}{r}3.00(0.45) \\
6.70(0.61) \\
13.10(1.21)\end{array}$ \\
\hline
\end{tabular}

$p \leq 0.005)$ and was inversely related to the total Parkinsonian disability score ( $p \leq 0.05$, table).

\section{Reaction time}

Simple RT was prolonged in the patients with $P D$ at all three infusion rates compared with the RT of normal controls ( $p \leq 0.01$, figure $1 a$, $t$ tests). There was no change in simple RT in relation to the plasma concentration of levodopa. The patients' choice RT was also significantly longer than simple RT for normal subjects ( $p \leq 0.0001$ ). There was no difference between choice and simple RT in PD patients For normal subjects, choice RT was longer than simple RT ( $p \leq 0.0001)$. Choice RT for the patients with PD was also longer than the choice RT of normal subjects at all infusion levels. Choice RT did not change with concentration of levodopa. RT did not vary with movement amplitude for either the patients or the normal subjects in either the simple or choice task.

\section{Movement time}

In contrast to RT findings, plasma levodopa concentration affected MT of the PD patients ( $\mathrm{p} \leq 0.0001$, fig $1 \mathrm{~b}$ ). For the patients, MT was longer at the lower rate than at the mid or high rates of levodopa infusion ( $p \leq 0.0005$ ). MT was the same in the simple or choice task for both the normal subjects and patients with PD.

Figure 1 Performance during simple and choice reaction time tasks. A Reaction time for normal controls $(N)$ and $P D$ patients. $R T$ was greater for the patients than for normal controls in both tasks. Simple RT was faster than choice $R T$ in normals, but not different from choice $R T$ in the patients. The plasma levodopa level did not affect $R T$ during either task. B Movement time for normal controls $(N)$ and $P D$ patients. $M T$ was typically greater than normal for the patients, though when optimally medicated the $P D$ patients moved nearly as fast as the normal controls. $M T$ for the patients slowed with decreases in available levodopa. Error bars are \pm one SEM for all statistics.

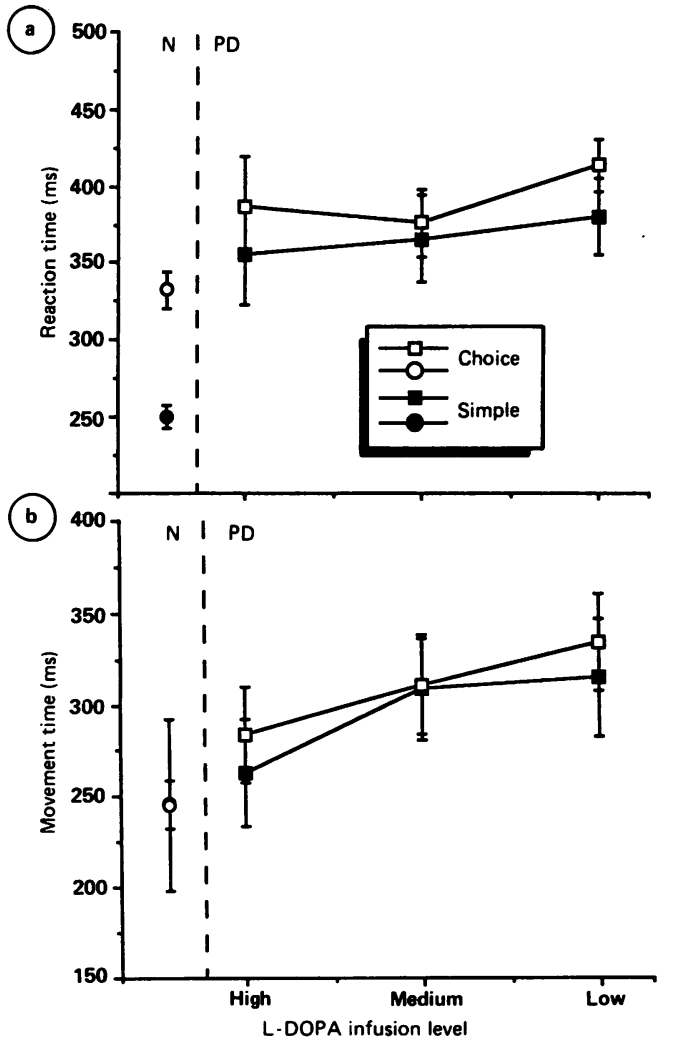

Additionally, MT for the patients at the high infusion level was indistinguishable from MT of the normal subjects. However, at the two lower rates of levodopa infusion, MT was longer for the $\mathrm{PD}$ patients compared with normal controls ( $\mathrm{p} \leq 0.01, t$ tests).

\section{Discussion}

RT deficits in PD patients are most evident when all movement parameters are fully prepared in advance, as in a simple RT task. ${ }^{51011}$ When specification of movement parameters is required concomitantly with movement initiation, as in a choice RT task, $\mathrm{PD}$ patients initiate movements either normally or with less of a "central" delay than normal subjects. ${ }^{51011}$ Recently, we found that choice RT of PD patients was sensitive to plasma, and presumably brain, levels of dopamine when movement direction and initiation were processed simultaneously. ${ }^{10}$ Our results suggest that in PD specification of movement amplitude, like simple movement initiation but in contrast to specification of movement direction, may not be sensitive to dopamine replacement therapy, and therefore may not be a dopaminergic process.

Behavioural and physiological studies have described distinctive coding for movement direction and amplitude. For example, the RT to begin movements with an unspecified direction is typically longer than for movements with an unspecified amplitude, ${ }^{78}$ thereby indicating some independence for the two processes. Additionally, the specification of direction and amplitude appear to be not only independent but also characteristically different. $^{22}{ }^{23}$ Both processes require about the same time, about $150 \mathrm{~ms}$, but have different forms. Amplitude specification begins with the selection of a presumed "default" mid-range amplitude, gradually becomes more accurate, and when enough time has elapsed, it is finally set with precision. ${ }^{22}$ In contrast, movement direction specification is a random process with subjects equally likely to choose one of the possible movement directions until the correct direction can be set after a sufficient time lapse. $^{23}$

Direct recordings of cerebral cortical cell firing suggest additional segregation of movement direction and amplitude coding. Cells in the primary motor and somatosensory cortices have discharges related to both movement direction (or position) and amplitude (or force)..$^{24-26}$ This may imply that the motor and sensory cortices are not primarily involved in preferentially specifying one or the other dimension of movement. In contrast, the parietal lobe, specifically area 5 , has cells with discharges that are solely related to movement direction and presumably independent of movement amplitude. ${ }^{27}$ Unfortunately, the supplementary motor area, the cortical target of basal ganglia output ${ }^{28}$ has not been studied with similar analyses regarding its coding for specification of movement amplitude.

It appears that the basal ganglia is also 
involved in processing information related to both the direction and amplitude of movement. For example, cellular discharge in several basal ganglia structures is related specifically to either the intended ${ }^{13}$ or the executed ${ }^{12}{ }^{14}$ direction of movement, and the discharge of some cells is related to the magnitude of muscle activity in a single direction. ${ }^{12}$ Thus, the relationship of basal ganglia neurons to applied loads is relatively weak, possibly indicating that the basal ganglia is not primarily involved in specifying the amount of muscle activity.

The deficit by PD patients in directional specification of movement has been examined in $R T$ tasks when directional coding is required at movement onset. ${ }^{10}$ In these studies, movement initiation is prolonged when subjects must process directional information concurrently with movernent initiation. In other studies, involving assessment of multiple segment movements for which changes in direction were required between the segments, Berardelli et $a l^{29}$ reported that PD patients paused excessively long between individual segments of a sequential two-dimensional movement. It might appear from these data that changing direction during movement execution, like $R T$, is also slowed in PD. However, this deficit may not be related only to the requirement to change direction, but instead may be due to the sequential nature of the task. For example, Benecke et al ${ }^{30}$ demonstrated that the time to begin the second of two independent movements executed sequentially was delayed in PD. Moreover, the inter-onset latency between the independent movements was dopamine sensitive. ${ }^{31}$ In their aggregate, these deficits were interpreted as indicating difficulty in selecting motor commands for sequential movements by PD patients. It may not be possible to generalise from the deficits in execution to deficits in initiating sequential movements, since Rafal et $a l^{6}$ showed that RT is unimpaired in $P D$ when a sequential movement task is being prepared. Thus, there may be a difference in directional processing when the sequential movement is prepared compared with when it is executed. Since the movements in the Berardelli ${ }^{29}$ study required changes in movement direction, it is possible that instead of reflecting a generalised deficit in motor programming, the excessive pauses between movement segments were related to a slowing of processing directional information, especially in a sequential motor task, in PD. This issue and the dopaminergic sensitivity of such behaviour were not evaluated and need further clarification.

In addition to reports of problems with directional coding in PD, there have been numerous investigations on whether magnitude coding is impaired in PD. Flowers ${ }^{32}$ noted that movements of $P D$ patients are typically hypometric when they are executed rapidly, especially for movements of larger amplitude. In many patients, the entire targeted movement is performed in several segments. Successive bursts of EMG activity accompany this segmented movement, ${ }^{1733}$ suggesting that PD patients have difficulty in emitting large amounts of muscle activity required to make large movements. The slow build up in the EMG during voluntary movement that is often observed in $\mathrm{PD}^{34}$ would support the notion that the mechanism controlling EMG magnitude in rapid movements is impaired in PD. Data obtained from performance on isometric tasks also indicate that PD patients have a slow EMG build up, but nevertheless ultimately achieve the correct end point. ${ }^{35}$ In contrast to these data on abnormalities in voluntary EMG responses are recent results showing that $P D$ patients can scale the size of the first agonist EMG burst when they perform movements of different magnitudes and against different loads. ${ }^{17} \mathrm{Al}$ though the magnitude of the EMG burst was diminished when PD patients were off medication, this study showed that the amplitude of the bursts in relation to intended movement size and opposing load was not altered by withdrawal of dopamine replacement therapy. ${ }^{17}$

These results indicate that the concurrent planning of movement amplitude and initiation by $P D$ patients is insensitive to plasma, and likely brain, levels of dopamine. This contrasts with our earlier data concerning directional choice $\mathrm{RT}^{10}$ and seemingly with results from other studies indicating that some aspects of amplitude specification are affected by Parkinsonism and sensitive to dopaminergic replacement therapy. ${ }^{1732} 34$ Nevertheless, we did find that the amplitude choice RT of patients was significantly slower than normal, even at the optimal levodopa infusion rate, indicating that Parkinsonism affects the concurrent specification of movement amplitude and initiation, though not necessarily through a dopaminergic mechanism.

1 Wilson SAK. Disorders of motility and of muscle tone, with special reference to the corpus striatum. Lancet 1925;ii: $1-10,53-62,169-78,215-9,268-76$.

2 Velasco F, Velasco $M$. A quantitative evaluation of the effects of L-dopa on Parkinson's disease. Neuropharmacology 1973;12:89-99.

3 Heilman KM, Bowers D, Watson RT, Greer M. Reaction times in Parkinson's disease. Arch Neurol 1976;33:139-40.

4 Evarts EV, Teräväinen H, Calne DB. Reaction time in Parkinson's disease. Brain 1981;104:167-86.

5 Stelmach GE, Worringham CJ, Strand EA. Movement preparation in Parkinson's disease. The use of advance information. Brain 1986;109:1179-94.

6 Rafal RD, Winhoff AW, Friedman JH, Bernstein E. Programming and execution of sequential movements in Parkinson's disease. J Neurol Neurosurg Psychiatry 1987; 50:1267-73.

7 Rosenbaum DA. Human movement initiation: specification of arm, direction, and extent. J Exp Psychol Gen 1980; 109:444-74.

8 Goodman D, Kelso JAS. Are movements prepared in parts? Not under compatible (naturalized) conditions. J Exp Psychol Gen 1980;109:475-95.

9 Sanes JN. Information processing deficits in Parkinson's disease during movement. Neuropsychologia 1985;23: 381-92.

10 Pullman SL, Watts RL, Juncos JL, Chase TN, Sanes JN. Dopaminergic effects on simple and choice reaction time performance in Parkinson's Disease. Neurology 1988, 38:249-54.

11 Bloxham CA, Mindel TA, Frith CD. Initiation and execution of predictable and unpredictable movements in Parkinson's disease. Brain 1984;107:371-84.

12 Crutcher MD, DeLong MR. Single cell studies of the primate putamen. I. Functional organization. Exp Brain Res 1984;53:233-43.

13 Alexander GE. Selective neuronal discharge in monkey putamen reflects intended direction of planned limb movements. Exp Brain Res 1987;67:623-34.

14 Mitchell SJ, Richardson RT, Baker FH, DeLong MR. The primate globus pallidus: neuronal activity related to 15 DeLong MR, Crutcher MD, Georgopoulos AP. Primate 
globus pallidus and subthalamus nucleus: functional organization. J Neurophysiol 1987;53:530-43.

16 Alexander GE, DeLong MR. Microstimulation of the primate neostriatum. II. Somatotopic organization of microexcitable zones and their relation to neuronal resmicroexcitable zones and their relation to neuronal

17 Berardelli A, Dick JPR, Rothwell JC, Day BL, Marsden CD. Scaling of the size of the first agonist EMG burst during rapid wrist movements in patients with Parkinson's disease. J Neurol Neurosurg Psychiatry 1986; 49:1273-9.

18 Hoehn MM, Yahr MD. Parkinsonism: onset, progression, and mortality. Neurology 1967;17:427-44.

19 Duvoisin RC. The evaluation of extrapyramidal disease. In: Ajuriaguerra J, Gauthier G, eds. Monoamines, noyaux gris centraux et syndrom de Parkinson. Paris: Masson, 1971: centraux

20 Wagner J, Vitali P, Pal-Freyman MG, Zraika M, Huot S. Simultaneous determination of 3,4-dihydroxyphenylalanine, 5-hydroxytryptophan, dopamine, 4-hydroxy-3methoxyphenylalanine, norepinephrine, 3,4-dihydroxymethoxyphenylalanine, norepinephrine, 3,4-dihydroxyphenylacetic acid, homovanillic acid, serotonin, and 5 -hydroxyindolacetic acid in rat cerebrospinal fluid and
brain by high performance liquid chromatography with brain by high performance liquid chromatography with
electrochemical detection. $J$ Neurochem 1982;38: 1241-54.

21 Sanes JN. Kinematics and end-point control of arm movements are modified by unexpected changes in viscous loading. $J$ Neurosci 1986;6:3120-7.

22 Favilla $M$, Hening $W$, Ghez $C$. Trajectory control in targeted force impulses. VI. Independent specification of response amplitude and direction. Exp Brain Res 1989; response ampl

23 Bermejo R, Pullman S, Ghez C. Differences in programming of amplitude and direction in a two dimensional force aiming task. Neurosci Abstr 1989;15:50.

24 Jennings VA, Lamour Y, Solis H, Fromm C. Somatosensory cortex activity related to position and force. $J$ Neurophysiol 1983;49:1216-29.
25 Schwartz AB, Kettner RE, Georgopoulos AP. Primate motor cortex and free arm movements to visual targets in discharge and direction of movement. $J$ Neurosci 1988; 8:2913-27.

26 Kalaska JF, Cohen DAD, Hyde ML, Prud'homme M. A comparison of movement direction-related versus load direction-related activity in primate motor cortex, using a two-dimensional reaching task. $J$ Neurosci 1989;9: 2080-102.

27 Kalaska JF. The representation of arm movements in postcentral and parietal cortex. Can J Physiol Pharmacol 1988;66:455-63.

28 Schell GR, Strick PL. The origin of thalamic inputs to the arcuate premotor and supplementary motor areas. $J$ Neurosci 1984;4:539-60.

29 Berardelli A, Accornero N, Argenta M, Meco G, Manfredi $M$. Fast complex arm movements in Parkinson's disease. M. Fast complex arm movements in Parkinson's

30 Benecke R, Rothwell JC, Dick JPR, Day BL, Marsden CD. Disturbance of sequential movements in patients with Parkinson's disease. Brain 1987;110:361-79.

31 Benecke R, Rothwell JC, Dick JPR, Day BL, Marsden CD. Simple and complex movements off and on treatment in patients with Parkinson's disease. J Neurol Neurosurg Psychiatry 1987;50:296-303.

32 Flowers KA. Visual 'closed-loop' and 'open-loop' characteristics of voluntary movement in patients with parkinsonism and intention tremor. Brain 1976;99:269-310.

33 Hallett $M$, Koshbin S. A physiological mechanism of bradykinesia. Brain 1980;103:301-14.

34 Evarts EV, Teräväinen HT, Beuchert BE, Calne DB. Pathophysiology of motor performance in Parkinson's Pathophysiology of motor performance in Parkinson's Derivative and Motor Function. Oxford: Pergamon Press, Derivative and
$1979: 45-59$.

35 Stelmach GE, Worringham CJ. The preparation and production of isometric force in Parkinson's disease. Neuropsychol 1988;26:93-103. 